\title{
Responsibility in Investigations on Human Subjects
}

\section{Statement by Medical Research Council}

\author{
Under this heading the Medical Research Council include in their Annual Report for 1962-63 (Cmnd. 2382, H.M.S.O., \\ 1964, price 16s. 6d. net) a statement on investigations on human beings which is intended to serve as a guide to \\ medical men engaged in this kind of work. It is a statement of such importance that we reproduce it here in full.
}

\begin{abstract}
During the last 50 years medical knowledge has advanced more rapidly than at any other period in its history. New understandings, new treatments, new diagnostic procedures, and new methods of prevention have been, and are being, introduced at an ever-increasing rate; and if the benefits that are now becoming possible are to be gained these developments must continue.

Undoubtedly the new era in medicine upon which we have now entered is largely due to the marriage of the methods of science with the traditional methods of medicine. Until the turn of the century the advancement of clinical knowledge was in general confined to that which could be gained by observation, and means for the analysis in depth of the phenomena of health and disease were seldom available. Now, however, procedures that can safely, and conscientiously, be applied to both sick and healthy human beings are being devised in profusion, with the result that certainty and understanding in medicine are increasing apace.
\end{abstract}

Yet these innovations have brought their own problems to the clinical investigator. In the past, the introduction of new treatments or investigations was infrequent, and only rarely did they go beyond a marginal variation on established practice. To-day far-ranging new procedures are commonplace, and such are their potentialities that their employment is no negligible consideration. As a result, investigators are frequently faced with ethical and sometimes even legal problems of great difficulty. It is in the hope of giving some guidance in this difficult matter that the Medical Research Council issue this statement.

A distinction may legitimately be drawn between procedures undertaken as part of patient-care which are intended to contribute to the benefit of the individual patient, by treatment, prevention, or assessment, and those procedures which are undertaken either on patients or on healthy subjects solely for the purpose of contributing to medical knowledge and are not themselves designed to benefit the particular individual on whom they are performed. The former fall within the ambit of patient-care and are governed by the ordinary rules of professional conduct in medicine; the latter fall within the ambit of investigations on volunteers.

Important considerations flow from this distinction.

\section{Procedures Contributing to the Benefit of the Individual}

In the case of procedures directly connected with the management of the condition in the particular individual, the relationship is essentially that between doctor and patient. Implicit in this relationship is the willingness on the part of the subject to be guided by the judgment of his medical attendant. Provided, therefore, that the medical attendant is satisfied that there are reasonable grounds for believing that a particular new procedure will contribute to the benefit of that particular patient, either by treatment, prevention, or increased understanding of his case, he may assume the patient's consent to the same extent as he would were the procedure entirely established practice. It is axiomatic that no two patients are alike and that the medical attendant must be at liberty to vary his procedures according to his judgment of what is in his patients' best interests. The question of novelty is only relevant to the extent that in reaching a decision to use a novel procedure the doctor, being unable to fortify his judgment by previous experience, must exercise special care. That it is both considerate and prudent to obtain the patient's agreement before using a novel procedure is no more than a requirement of good medical practice.

The second important consideration that follows from this distinction is that it is clearly within the competence of a parent or guardian of a child to give permission for procedures intended to benefit that child when he is not old or intelligent enough to be able himself to give a valid consent.

A category of investigation that has occasionally raised questions in the minds of investigators is that in which a new preventive, such as a vaccine, is tried. Necessarily, preventives are given to people who are not, at the moment, suffering from the relevant illness. But the ethical and legal considerations are the same as those that govern the introduction of a new treatment. The intention is to benefit an individual by protecting him against a future hazard; and it is a matter of professional judgment whether the procedure in question ofiers a better chance of doing so than previously existing measures.

In general, therefore, the propriety of procedures intended to benefit the individual-whether these are directed to treatment, to prevention, or to assessment-are determined by the same considerations as govern the care of patients. At the frontiers of knowledge, however, where not only are many procedures novel but their value in the particular instance may be debatable, it is wise, if any doubt exists, to obtain the opinion of experienced colleagues on the desirability of the projected procedure.

\section{Control Subjects in Investigations of Treatment or Prevention}

Over recent years the development of treatment and prevention has been greatly advanced by the method of the controlled clinical trial. Instead of waiting, as in the past, on the slow accumulation of general experience to determine the relative advantages and disadvantages of any particular measure, it is now often possible to put the question to the test under conditions which will not only yield a speedy and more precise answer but also limit the risk of untoward effects remaining undetected. Such trials are, however, only feasible when it is possible to compare suitable groups of patients and only permissible when there is a genuine doubt within the profession as to which of the two treatments or preventive regimes is the better. In these circumstances it is justifiable to give to a proportion of the patients the novel procedure on the understanding that the remainder receive the procedure previously accepted as the best. In the case when no effective treatment has previously been devised then the situation should be fully explained to the participants and their true consent obtained.

Such controlled trials may raise ethical points which may be of some difficulty. In general, the patients participating in them should be told frankly that two different procedures are being assessed and their co-operation invited. Occasionally, however, to do so is contraindicated. For example, to awaken patients with a possibly fatal illness to the existence of such doubts about effective treatment may not always be in their best interest; or suspicion may have arisen as to whether a 
particular treatment has any effect apart from suggestion, and it may be necessary to introduce a placebo into part of the trial to determine this. Because of these and similar difficulties it is the firm opinion of the Council that controlled clinical trials should always be planned and supervised by a group of investigators and never by an individual alone. It goes without question that any doctor taking part in such a collective controlled trial is under an obligation to withdraw a patient from the trial, and to institute any treatment he considers necessary, should this, in his personal opinion, be in the better interests of his patient.

\section{Procedures not of Direct Benefit to the Individual}

The preceding considerations cover the majority of clinical investigations. There remains, however, a large and important field of investigations on human subjects which aim to provide normal values and their variation so that abnormal values can be recognized. This involves both ill persons and "healthy" persons, whether the latter are entirely healthy or patients suffering from a condition that has no relevance to the investigation. In regard to persons with a particular illness, such as metabolic defect, it may be necessary to know the range of abnormality compatible with the activities of normal life or the reaction of such persons to some change in circumstances, such as an alteration in diet. Similarly it may be necessary to have a clear understanding of the range of a normal function and its reaction to changes in circumstances in entirely healthy persons. The common feature of this type of investigation is that it is of no direct benefit to the particular individual and that, in consequence, if he is to submit to it he must volunteer in the full sense of the word.

It should be clearly understood that the possibility or probability that a particular investigation will be of benefit to humanity or to posterity would afford no defence in the event of legal proceedings. The individual has rights that the law protects and nobody can infringe those rights for the public good. In investigations of this type it is therefore always necessary to ensure that the true consent of the subject is explicitly obtained.

By true consent is meant consent freely given with proper understanding of the nature and consequences of what is proposed. Assumed consent or consent obtained by undue influence is valueless, and, in this latter respect, particular care is necessary when the volunteer stands in special relationship to the investigator, as in the case of a patient to his doctor, or a student to his teacher.

The need for obtaining evidence of consent in this type of investigation has been generally recognized, but there are some misunderstandings as to what constitutes such evidence. In general, the investigator should obtain the consent himself in the presence of another person. Written consent unaccompanied by other evidence that an explanation has been given, understood, and accepted, is of little value.

The situation in respect of minors and mentally subnormal or mentally disordered persons is of particular difficulty. In the strict view of the law parents and guardians of minors cannot give consent on their behalf to any procedures which are of no particular benefit to them and which may carry some risk of harm. Whilst English law does not fix any arbitrary age in this context, it may safely be assumed that the Courts will not regard a child of 12 years or under (of 14 years or under for boys in Scotland) as having the capacity to consent to any procedure which may involve him in an injury. Above this age the reality of any purported consent which may have been obtained is a question of fact, and as with an adult the evidence would, if necessary, have to show that irrespective of age the person concerned fully understood the implications to himself of the procedures to which he was consenting.

In the case of those who are mentally subnormal or mentally disordered the reality of the consent given will fall to be judged by similar criteria to those which apply to the making of a will, contracting a marriage, or otherwise taking decisions which have legal force as well as moral and social implications. When true consent in this sense cannot be obtained, procedures which are of no direct benefit and which might carry a risk of harm to the subject should not be undertaken.

Even when true consent has been given by a minor or a mentally subnormal or mentally disordered person, considerations of ethics and prudence still require that, if possible, the assent of parents or guardians or relatives, as the case may be, should be obtained.

Investigations that are of no direct benefit to the individual require, therefore, that his true consent to them shall be explicitly obtained. After adequate explanation, the consent of an adult of sound mind and understanding can be relied upon to be true consent. In the case of children and young persons the question whether purported consent was true consent would in each case depend upon facts such as the age, intelligence, situation, and character of the subject, and the nature of the investigation. When the subject is below the age of 12 years, information requiring the performance of any procedure involving his body would need to be obtained incidentally to and without altering the nature of a procedure intended for his individual benefit.

\section{Professional Discipline}

All who have been concerned with medical research are aware of the impossibility of formulating any detailed code of rules which will ensure that irreproachability of practice which alone will suffice where investigations on human beings are concerned. The law lays down a minimum code in matters of professional negligence and the doctrine of assault. But this is not enough. Owing to the special relationship of trust that exists between a patient and his doctor, most patients will consent to any proposal that is made. Further, the considerations involved in a novel procedure are nearly always so technical as to prevent their being adequately understood by one who is not himself an expert. It must therefore be frankly recognized that, for practical purposes, an inescapable moral responsibility rests with the doctor concerned for determining what investigations are, or are not, proposed to a particular patient or volunteer. Nevertheless, moral codes are formulated by man, and if, in ever-changing circumstances of medical advance, their relevance is to be maintained, it is to the profession itself that we must look, and in particular to the heads of departments, the specialized Societies, and the editors of medical and scientific journals.

In the opinion of the Council, the head of a department where investigations on human subjects take place has an inescapable responsibility for ensuring that practice by those under his direction is irreproachable.

In the same way the Council feel that, as a matter of policy, bodies like themselves that support medical research should do everything in their power to ensure that the practice of all workers whom they support shall be unexceptionable and known to be so.

So specialized has medical knowledge now become that the profession in general can rarely deal adequately with ind:vidual problems. In regard to any particular type of investigation, only a small group of experienced men who have specialized in this branch of knowledge are likely to be competent to pass an opinion on the justification for undertaking any particular procedure. But in every branch of medicine specialized scientific societies exist. It is upon these that the profession in general must mainly rely for the creation and maintenance of that body of precedents which shall guide individual investigators in case of doubt, and for the critical discussion of the communications presented to them on which the formation of the necessary climate of opinion depends. 
Finally, it is the Council's opinion that any account of investigations on human subjects should make clear that the appropriate requirements have been fulfilled, and, further, that no paper should be accepted for publication if there are any doubts that such is the case.

The progress of medical knowledge has depended, and will continue to depend, in no small measure upon the confidence which the public has in those who carry out investigations on human subjects, be these healthy or sick. Only in so far as it is known that such investigations are submitted to the highest ethical scrutiny and self-discipline will this confidence be maintained. Mistaken, or misunderstood, investigations could do incalculable harm to medical progress. It is our collective duty as a profession to see that this does not happen and so to continue to deserve the confidence that we now enjoy.

\section{Work of Medical Research Council}

The Report of the Medical Research Council for 1962-63 (Cmnd. 2382, H.M.S.O., 1964, price 16s. 6d. net) contains a review of topics of current interest in the medical and biological sciences which have been under study during the year. The following is a short summary of some of the topics discussed.

\section{Organ Transplantation}

The report notes that public interest in the surgical transplantation of organs has been aroused as the result of some successes in kidney transplantation, and discusses some of the theoretical principles involved. The first necessary condition for a successful transplant is that the organ must be able to survive for a period without a blood supply, and the second is that it must be capable of functioning adequately in the absence of nervous control. Even when these conditions are fulfilled, homotransplants are still usually destroyed after a few weeks, and it is now clear that this is an immunological phenomenon. There appear to be two main types of immunological reaction, the first of which involves antibodies, and the second being mediated by cells in the absence of antibodies. The particular antigens present in a homotransplant which evoke the reaction are determined by the genetic make-up of the donor.

When homotransplants survive, it is because there are no antigens in the transplant which are lacking in the recipient, as in identical twins. Clinically, efforts are made to reduce the recipient's capacity for immunological response by $x$-rays or by the administration of antimetabolic drugs. An experimental procedure which has no direct clinical application but is of great theoretical importance is the induction in animals of specific immunological tolerance by injecting the animal during embryonic life or immediately after birth with living cells from an older animal of the same species. This occurs naturally when non-identical twins interchange blood-forming tissue during foetal life. More recently it has been found possible to induce specific tolerance in adult animals by injecting cells after exposing them to $x$-rays.

So far, at least 25 patients have been successfully treated with renal transplantation. Unfortunately irradiation and the administration of antimetabolic drugs have the effect of reducing resistance to infection, and the failure rate of the operation is high, but several patients are live and well more than a year after the operation. While some of these patients are still receiving antimetabolic drugs, others have proved able to survive without them, and it may be that in these patients specific tolerance has developed.
It appears that there is individual variation in the way patients react, and it is suggested that there may be two possible lines of future advance : the development of methods for selecting compatible donors, and an improvement in the methods used to diminish the recipient's capacity to react against his transplant.

\section{Clinical Geneties}

While the remarkable work of recent years in molecular biology may have to some extent overshadowed the more practical applications of clinical genetics, the advances in this field are nevertheless substantial. There are a number of genetic disorders for which prompt and effective treatment can be given if they are recognized early enough. An example of this is phenylketonuria, which can be modified by diet if treated at an early age. The same applies to galactosaemia, a condition dependent on a recessive gene in which an essential enzyme for the breakdown of lactose is absent. If lactose is withheld from the diet the accumulation of toxic products can be prevented and normal or almost normal development assured. Recognition is important, not simply for the individual, but for other members of the family. Other genetic diseases are nephrogenic diabetes insipidus, which may lead to mental deficiency, and multiple polyposis of the colon and rectum, which is a pre-cancerous condition.

An example of the clinical application of genetics is the family study of glaucoma simplex by Miller and Patterson at Moorfields Eye Hospital. These workers found that only four out of 50 sibs of affected persons were similarly affected, and only two out of 75 children. However, when a coefficient of the outflow of the fluid from the anterior chamber of the eye was estimated, it was found that about half the sibs and half of the children showed abnormal values, as against practically none of the controls. There are similar applications of genetic principles in the field of drug-induced conditions, where sensitivities to otherwise safe and useful drugs have been found to have a genetic basis. The condition of hereditary porphyria which has been described by Dean in South Africa is due to a dominant gene and is transmitted with perfect regu- larity from affected persons to half their children on the average. These patients show a sensitivity of the skin and great idiosyncrasy to certain drugs, particularly the barbiturates.

In the present state of knowledge the most important application of genetics is the provision of advice for those who need it, particularly parents who may be taking the risk of having abnormal children. Of particular importance is genetic advice in relation to mental diseases, and research in this subject is being carried out by the Council's Psychiatric Genetics Research Unit at the Maudsley Hospital.

However, defects due to simple inheritance are rare, though heredity as a factor is much more common. One factor making for complication is that the same end result may be due to different genes. One of the reasons why simple inherited defects are rare is that mutation is rare and the processes of natural selection ensure that ordinarily the rate of manifestation of such defects is kept down to something near the mutation rate. This is not invariable, however. One notable exception is sickle-cell anaemia in Africa, which actually confers protection against malignant tertian malaria.

Most common congenital malformations have a hereditary element in their causation, but it is not a simple one and non-genetic factors are also involved. It is consequently difficult to assess the risk to relatives as compared with the general population. It is usual now to regard multifactorial inheritance, depending on the combined action of many genes, as the most likely explanation for many of these diseases. A general review of multifactorial inheritance in relation to human disease has recently been published by Roberts (1964), Director of the Council's Clinical Genetics Research Unit

\section{Harmful Effects of Radiation}

It is well known that heavy doses of radiation, particularly to the whole body, can produce a severe depression of the bonemarrow, and that a major contributing cause of such effects is severe chromosome damage at the time of radiation. A more difficult problem is the extent to which chromosome damage may play a part in the late effects of exposure, particularly the development of cancers. It is now accepted that exposure to heavy doses of radiation increases the risk of leukaemia and that exposure of the unborn child to low levels of radiation during the course of $x$-ray examination of the mother's abdomen or pelvis increases the risk of leukaemia during early childhood. It is possible that this may be due to the production of genetic damage in a progenitor 